\title{
Multiple Charge States of Ag Atoms on Ultrathin NaCl Films
}

\author{
F. E. Olsson, ${ }^{1}$ S. Paavilainen, ${ }^{2}$ M. Persson, ${ }^{3}$ J. Repp, ${ }^{4,5}$ and G. Meyer ${ }^{5}$ \\ ${ }^{1}$ Department of Applied Physics, Chalmers University of Technology, 41296 Göteborg, Sweden \\ ${ }^{2}$ Institute of Physics, Tampere University of Technology, 33720 Tampere, Finland \\ ${ }^{3}$ Surface Science Research Centre and Department of Chemistry, The University of Liverpool, Liverpool L69 3BX, United Kingdom \\ ${ }^{4}$ Institute of Experimental and Applied Physics, University of Regensburg, 93040 Regensburg, Germany \\ ${ }^{5}$ IBM Research, Zurich Research Laboratory, 8803 Rüschlikon, Switzerland
}

(Received 15 December 2006; published 25 April 2007)

\begin{abstract}
A combined study using density functional calculations and scanning tunneling microscopy experiments shows that individual silver adatoms on ultrathin sodium chloride films on copper surfaces are stable in three different charge states - neutral, negatively, and positively charged adatoms. The charge states of the individual adatoms were manipulated by voltage pulses. The key parameters determining the stability of various charge states are identified and discussed within a simple model.
\end{abstract}

DOI: 10.1103/PhysRevLett.98.176803

Recent progress in scanning tunneling microscopy (STM) experiments of adsorbates on ultrathin, insulating films has opened up a new field in atomic-scale science. A unique feature of these films is the reduction of the coupling of adsorbate electronic states with the metal support, which enabled the manipulation of electronic [1,2], magnetic [3,4], and conducting [5,6] properties of single adsorbates. A most interesting observation was the charge bistability of individual $\mathrm{Au}$ atoms on $\mathrm{NaCl}$ films [2] and the ability to switch controllably between these two different charge states. Recently, singly and doubly charged vacancies have been identified in ultrathin $\mathrm{MgO}$ films supported by an $\mathrm{Ag}$ substrate [7]. The existence and manipulation of multiple charge states of single adatoms will be important for the control of the current in molecular-scale memories and electronics [8]. Moreover, the study of these charging phenomena on an atomic level has ramifications for our understanding of local polaron formation, electron transfer, and electron solvation [9] in a condensed environment, as well as for catalysis on supported metal atom clusters [10].

So far, the observation and manipulation of different charge states of adsorbed atoms have been limited to $\mathrm{Au}$ atoms on $\mathrm{NaCl}$ films supported by a $\mathrm{Cu}$ substrate [2]. On ultrathin $\mathrm{MgO}$ films supported by a Mo substrate, density functional calculations suggested that Au adatoms should be spontaneously negatively charged [11]. Thus, it is important to clarify whether the charge multistability of adatoms is unique for the $\mathrm{Au} / \mathrm{NaCl} / \mathrm{Cu}$ system and limited to bistability. Furthermore, one needs to identify the key physical parameters behind this phenomenon.

In this Letter, we show from a combined STM and density functional calculations study that $\mathrm{Ag}$ atoms adsorbed on $\mathrm{NaCl}$ bilayers on $\mathrm{Cu}(100)$ exhibit charge tristability and can be switched reversibly between the neutral adatom state and the negatively or the positively charged adatom state. Thus, the charge multistability of adatoms is not at all unique for Au adatoms, and it is even possible to have more than two different charge states of an
PACS numbers: 73.20.Hb, 68.37.Ef, 71.15.Mb, 73.61.Ng

adatom. Furthermore, based on a simple model, we identify and discuss the key adatom, film, and substrate parameters for the stabilization of multiple charge states. This model can be used as a guide to find other systems of interest.

The experiments were carried out with a home-built low-temperature STM operated at $5 \mathrm{~K}$. $\mathrm{NaCl}$ was evaporated thermally onto clean copper single-crystals having (111), (100), and (311) surface orientations. Ag atoms were deposited inside the STM at a sample temperature below $10 \mathrm{~K}$. For the charge state switching experiments, we positioned the tip on top of an Ag adatom, retracted the tip by up to $8 \AA$, and applied a voltage pulse of different polarities with the feedback loop turned off. Here, all bias voltages refer to the sample voltage with respect to the tip so that a positive and a negative pulse corresponds to electron and hole tunneling from the tip to the sample, respectively. In the following, the notation $A^{q}$ refers to an adatom A with charge qe adsorbed on a $\mathrm{NaCl}$ film. The data refers to a bilayer $\mathrm{NaCl}(2 \mathrm{ML}) / \mathrm{Cu}(100)$, except where stated otherwise. The charge state of an $\mathrm{Ag}$ adatom was identified from (1) manipulation of the charge state by electron or hole tunneling, (2) characteristic STM images of the adatom, and (3) the observed behavior of adatom scattering of interface-state electrons on $\mathrm{NaCl}(2 \mathrm{ML}) /$ $\mathrm{Cu}(111)$.

Upon deposition, $\mathrm{Ag}$ atoms adsorb in a neutral adatom state $\mathrm{Ag}^{0}$. As shown in Fig. 1, the image of $\mathrm{Ag}^{0}$ is characterized by a wide featureless protrusion of about $2.5 \AA$ in height. A further analysis of the images, as detailed below, reveals that $\mathrm{Ag}^{0}$ always adsorbs on top of a $\mathrm{Cl}$ anion. However, all these adsorption sites are not equivalent because of the formation of Moire patterns by the slight lattice mismatch between the $\mathrm{NaCl}$ film and the $\mathrm{Cu}(100)$ surface [12].

Similarly to the $\mathrm{Au}$ adatom charge manipulation [2], $\mathrm{Ag}^{0}$ can be switched to a negatively charged adatom state $\mathrm{Ag}^{-}$by electron tunneling ( $\left.V \geqslant 1.3 \mathrm{~V}[13]\right)$. As for an $\mathrm{Au}$ 


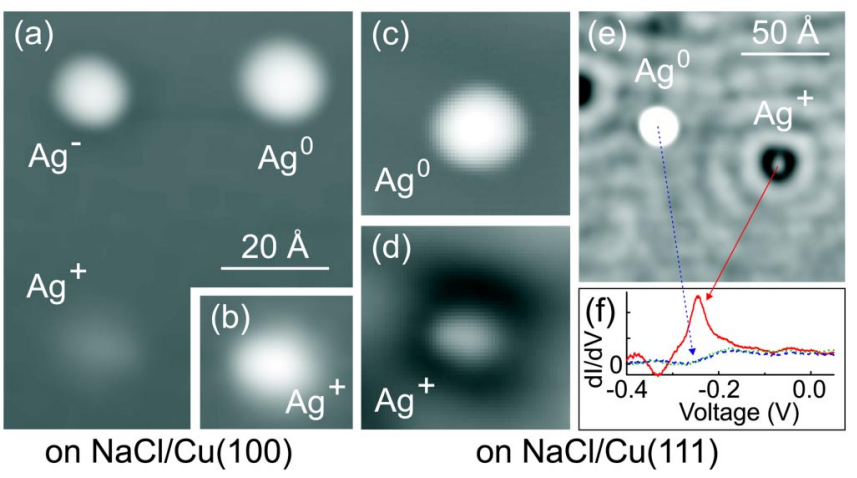

FIG. 1 (color online). STM images and spectra of different Ag adatom states $\left(\mathrm{Ag}^{0}, \mathrm{Ag}^{+}\right.$, and $\left.\mathrm{Ag}^{-}\right)$on $\mathrm{NaCl}(2 \mathrm{ML}) / \mathrm{Cu}(100)$ ((a) and (b)) and $\mathrm{Cu}(111)$ ((c) to (f)). In (a) and (c), the maximum scale (white) corresponds to a height of $2.5 \AA$; (b) and (d) show $\mathrm{Ag}^{+}$with the contrast being $4 \times$ higher. An image at even higher contrast (e) and local $d I / d V$ spectra (f) show scattering of interface-state electrons and an interface-state localization at $\mathrm{Ag}^{+}$but not at $\mathrm{Ag}^{0}$. The solid, dashed, and dotted spectrum refers to $\mathrm{Ag}^{+}, \mathrm{Ag}^{0}$ and bare $\mathrm{NaCl} / \mathrm{Cu}$, respectively. The bias and the tunneling currents are (a) $50 \mathrm{mV}, 1 \mathrm{pA}$; (b) $200 \mathrm{mV}, 0.5 \mathrm{pA}$; (c) $-558 \mathrm{mV}, 62 \mathrm{pA}$; (d) $-211 \mathrm{mV}$, $62 \mathrm{pA}$; (e) $15 \mathrm{mV}, 0.8 \mathrm{pA}$.

adatom, the formation of $\mathrm{Ag}^{-}$results in both a sudden drop in the current signal by about a factor of 3 and an image that is characterized by a $2.0-\AA$-high protrusion, surrounded by a faint depression (sombrero shape). The switching is also reversible, that is, $\mathrm{Ag}^{-}$can be transformed back into $\mathrm{Ag}^{0}$ by hole tunneling $(V \lesssim-0.2 \mathrm{~V}$ [13]). Note that because of the Moire pattern, $\mathrm{Ag}^{-}$is unstable for some sites and will switch back to $\mathrm{Ag}^{0}$ spontaneously before $\mathrm{Ag}^{-}$can be imaged. On a trilayer $\mathrm{NaCl}(3 \mathrm{ML}) / \mathrm{Cu}(100)$, this spontaneous neutralization always occurs.

In contrast to an $\mathrm{Au}$ adatom, an $\mathrm{Ag}$ adatom can also be manipulated to a positively charged adatom state $\mathrm{Ag}^{+}$by hole tunneling ( $V \lesssim-1.3 \mathrm{~V}$ [13]). This manipulation is possible for all adsorption positions of $\mathrm{Ag}^{0}$ with respect to the Moiré pattern and results in a stable $\mathrm{Ag}^{+}$. The images of an $\mathrm{Ag}^{+}$is characterized by a small protrusion of only about $0.5 \AA$ in height and having an elongated shape. By electron tunneling ( $V \gtrsim 1.5 \mathrm{~V}[13]), \mathrm{Ag}^{+}$can again be neutralized but also be desorbed.

The adsorption sites of $\mathrm{Ag}^{0}, \mathrm{Ag}^{-}$and $\mathrm{Ag}^{+}$were determined experimentally from the STM images. In atomically resolved images [14-16], $\mathrm{Ag}^{+}$was found at a site bridging two $\mathrm{Cl}$ anions with its protrusion being elongated towards the two nearest neighboring $\mathrm{Cl}$ anions. Using the $\mathrm{Ag}^{+}$as a marker, the adsorption sites of $\mathrm{Ag}^{-}$and $\mathrm{Ag}^{0}$ were both found to be on top of the $\mathrm{Cl}$ anions.

To provide further justification of our assignment of the charge states of the different $\mathrm{Ag}$ adatom states, we employed a recently developed scheme [5] based on the observed behavior of the interaction of interface-state (IS) electrons of $\mathrm{NaCl} / \mathrm{Cu}(111)$ with the $\mathrm{Ag}$ adatoms. On this surface, the $\mathrm{Ag}$ adatoms exhibit a similar switching behavior as on $\mathrm{NaCl} / \mathrm{Cu}(100)$ except that $\mathrm{Ag}^{-}$is not stable, which we attribute to the larger work function of $\mathrm{NaCl} / \mathrm{Cu}(111)$. The identification of a localized IS in the $d I / d V$ spectrum (Fig. 1(f)) at $\mathrm{Ag}^{+}$shows that it is positively charged $[17,18]$. In the case of $\mathrm{Ag}^{0}$, both the absence of a localized IS and a much weaker standing-wave pattern of IS electrons than from $\mathrm{Ag}^{+}$are consistent with $\mathrm{Ag}^{0}$ being neutral (Figs. 1(e) and 1(f)). Finally, the similarities of the manipulation procedure and STM images of $\mathrm{Ag}^{-}$to $\mathrm{Au}^{-}$[2] indicate that $\mathrm{Ag}^{-}$is negatively charged.

To gain physical insight into the nature of $\mathrm{Ag}$ adatom states on $\mathrm{NaCl}(2 \mathrm{ML}) / \mathrm{Cu}(100)$, we have carried out periodic density functional theory calculations of their geometric and electronic structure and energetics, using the VASP code $[19,20]$. The electron-ion core interactions were treated by the projector augmented wave method $[21,22]$. The electron exchange-correlation interactions were treated by a generalized gradient approximation (GGA) [23]. To avoid an unphysical fractional occupation of the $\mathrm{Ag}^{0}$ as is introduced by an underestimate of the integer discontinuities of the chemical potential in the GGA [24], we included also an on-site Coulomb repulsion $U=$ $1.6 \mathrm{eV}(\mathrm{GGA}+U)[25]$ in an $s$ projector of the $\mathrm{Ag}$ atom. The value of $U$ was chosen to reproduce the proper discontinuity of the free atom, as determined by its calculated affinity and ionization energies [26].

In our calculations, we have identified three different charge states of the $\mathrm{Ag}$ adatoms $-\mathrm{Ag}^{0}, \mathrm{Ag}^{+}$, and $\mathrm{Ag}^{-}-$ that are (meta-)stable and exhibit the same characteristics as $\mathrm{Ag}^{0}, \mathrm{Ag}^{+}$, and $\mathrm{Ag}^{-}$in the experiments. The adsorption site on top of the $\mathrm{Cl}$ anion is found to be the most stable site for the neutral $\mathrm{Ag}^{0}$ [27,28] and the negatively charged $\mathrm{Ag}^{-}$, whereas the adsorption site bridging two $\mathrm{Cl}$ anions is the most stable site for the positively charged $\mathrm{Ag}^{+} . \mathrm{Ag}^{+}$ and $\mathrm{Ag}^{0}$ were both found to be weakly bonded to the surface, with adsorption energies $\left(E_{a}\right)$ of 0.30 and $0.11 \mathrm{eV}$, respectively. Despite its small negative $E_{a}$ of about $-0.01 \mathrm{eV}$, the $\mathrm{Ag}^{-}$state is metastable owing to the formation of an energy barrier for desorption by the ionic relaxations as discussed below.

In our calculations, $\mathrm{Ag}^{+}$on top of a $\mathrm{Na}$ cation is only slightly less stable $\left(E_{a}=0.26 \mathrm{eV}\right)$ than $\mathrm{Ag}^{+}$in bridge position. The ionic relaxations pattern is similar to the one induced by $\mathrm{Ag}^{-}$when interchanging the $\mathrm{Na}$ cations and $\mathrm{Cl}$ anions in the film. This adsorption state of $\mathrm{Ag}^{+}$has not been found in the experiment. It could be that $\mathrm{Ag}^{+}$in bridge position is favored as a result of the trajectory in the switching of $\mathrm{Ag}^{0}$ on top of the $\mathrm{Cl}$ site to a positively charged state. In the following, we will only refer to the bridge $\mathrm{Ag}^{+}$adsorption state.

The bonding of the $\mathrm{Ag}^{-}$and $\mathrm{Ag}^{+}$to the $\mathrm{NaCl}$ film is qualitatively different from each other, as indicated by their different adsorption geometries. The $\mathrm{Ag}^{+}$has a short $\mathrm{Ag}$ $\mathrm{Cl}$ bond length of $2.3 \AA$ and is adsorbed only $0.5 \AA$ outside the nearest neighboring $\mathrm{Cl}$ anions, which have a large 




FIG. 2. Calculated PDOS around $\mathrm{Ag}^{0}, \mathrm{Ag}^{+}$and $\mathrm{Ag}^{-}$on $\mathrm{NaCl}(2 \mathrm{ML}) / \mathrm{Cu}(100)$. (a) PDOS of $s$ - and $p$-waves around $\mathrm{Ag}^{+}$and its nearest neighboring $\mathrm{Cl}$ atom, respectively. (b) PDOS of $s$-waves around $\mathrm{Ag}^{0}$ and $\mathrm{Ag}^{-}$. For $\mathrm{Ag}^{0}$ an onsite Coulomb repulsion of $U=1.6 \mathrm{eV}$ and spin polarization were used. Note that the broadening of PDOS is artificial and its intrinsic broadening cannot be resolved in these calculations.

outward relaxation by $0.5 \AA$. $\mathrm{Ag}^{-}$, on the other hand, has a large Ag-Cl interatomic distance of $3.5 \AA$ and induces large relaxations in the $\mathrm{NaCl}$ film: an outward and inward relaxation of about $0.6 \AA$ of the nearest neighboring $\mathrm{Na}$ cations and $\mathrm{Cl}$ anions, respectively. This relaxation pattern is similar to the one induced by the $\mathrm{Au}^{-}$adatom on top of an $\mathrm{Cl}$ anion. In contrast to the charged adatom states, the neutral $\mathrm{Ag}^{0}$ does not induce any significant relaxations of the $\mathrm{Na}$ cations and $\mathrm{Cl}$ anions, and adsorbs at a large $\mathrm{Ag}-\mathrm{Cl}$ interatomic distance of about $3.0 \AA$.

The charge states of $\mathrm{Ag}^{0}, \mathrm{Ag}^{+}$, and $\mathrm{Ag}^{-}$and the nature of the adatom-film bond are revealed by the calculated partial density of states (PDOS) (Fig. 2) and also by electron density rearrangements upon adsorption (Fig. 3). The PDOS of $s$-waves around the Ag adatom shows that the state derived from the singly occupied $5 s$-orbital of the free $\mathrm{Ag}$ atom is fully occupied for the $\mathrm{Ag}^{-}$, singly occupied for the $\mathrm{Ag}^{0}$, and unoccupied for $\mathrm{Ag}^{+}$. These findings corroborate our previous experimental assignment of the charge states of the Ag adatoms. The PDOS around the
$\mathrm{Ag}^{+}$adatom and the nearest neighboring $\mathrm{Cl}$ anion also exhibit some mixing of $\mathrm{Ag} s$-states with $\mathrm{Cl} p$-states corresponding to a depopulation of antibonding $\mathrm{Ag}-\mathrm{Cl}$ states and the formation of a $\left[\mathrm{AgCl}_{2}\right]^{-}$complex with a covalent character of the bonding of the $\mathrm{Ag}^{+}$and $\mathrm{Cl}^{-}$ions. This depopulation shows up as an accumulation of electron density between the Ag adatom and the nearest neighboring $\mathrm{Cl}$ anions (Fig. 3(a)). In contrast, the electron density difference for the $\mathrm{Ag}^{-}$(Fig. 3(c)) only shows a large electron accumulation around the adatom and the formation of an ionic bond.

The physical mechanism behind the stabilization of various charged adatom states is the formation of an energy barrier between an adatom with charge $q e= \pm e$ and the neutral adatom by ionic relaxations in the film (Fig. 4). This diabatic picture is similar to the classical Marcus picture for electron transfer [29]. A necessary condition for the existence of such an energy barrier is the condition that the energy costs $\Delta E(0 \rightarrow q)$ and $\Delta E(q \rightarrow 0)$ for charging the neutral adatom $A^{0}$ and neutralizing the charged adatom $A^{q}$ at fixed equilibrium ionic configurations, respectively, both be positive. The key parameters determining these energies are most easily identified by assuming that the ionic and the electronic response of the film and the metal substrate are linear to the charging of the adatom. Under this assumption, the charge-switching energybarriers are given by

$$
\begin{gathered}
\Delta E(0 \rightarrow q)=\left\{\begin{array}{ll}
\Phi-A-\Delta E_{\mathrm{el}}, & \text { for } q=-1 \\
I-\Phi-\Delta E_{\mathrm{el}}, & \text { for } q=+1
\end{array},\right. \\
\Delta E(q \rightarrow 0)=2 \Delta E_{\mathrm{ion}}-\Delta E(0 \rightarrow q),
\end{gathered}
$$

where $A$ and $I$ are the affinity and ionization energies of the free atom, respectively, and $\Delta E_{\mathrm{el}}$ and $\Delta E_{\text {ion }}$ are the electronic relaxation and ionic reorganization energies gained by charging the atom, respectively, and $\Phi$ is the work function of the surface. A large $\Delta E_{\text {ion }}$ favor the stabilization of $A^{q}$ and is obtained whenever the static dielectric constant is substantially larger than the optical one for the film.

There is a delicate balance between the other key parameters $A, I, \Phi$, and $\Delta E_{\mathrm{el}}$ for the stability of the various

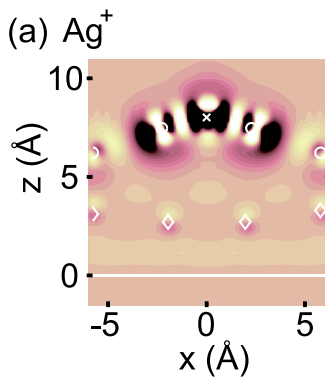

(b) $\mathrm{Ag}^{0}$
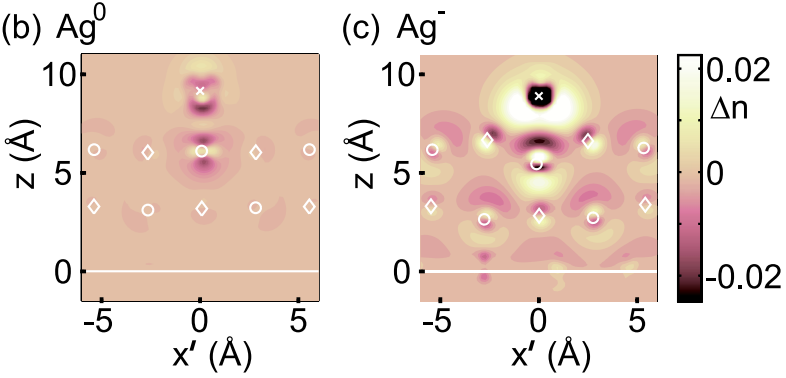

FIG. 3 (color online). Cross section of the calculated electron density differences, $\Delta n$, of (a) $\mathrm{Ag}^{+}$, (b) $\mathrm{Ag}^{0}, \mathrm{and}$ (c) $\mathrm{Ag}^{-} . \Delta n=$ $n_{\mathrm{Ag}+\text { substr }}-n_{\mathrm{Ag}}-n_{\text {substr }}$, given in units of $e / \AA^{3}$. The positions of the $\mathrm{Ag}$ atoms, $\mathrm{Na}$, and $\mathrm{Cl}$ ions are represented by crosses, diamonds, and circles, respectively. The $\mathrm{Cu}(100)$ surface plane is indicated by a solid line. $x$ and $x^{\prime}$ are the coordinates along the [011] (a) and [100] $(b, c)$ direction of the $\mathrm{NaCl}$ film, respectively. 


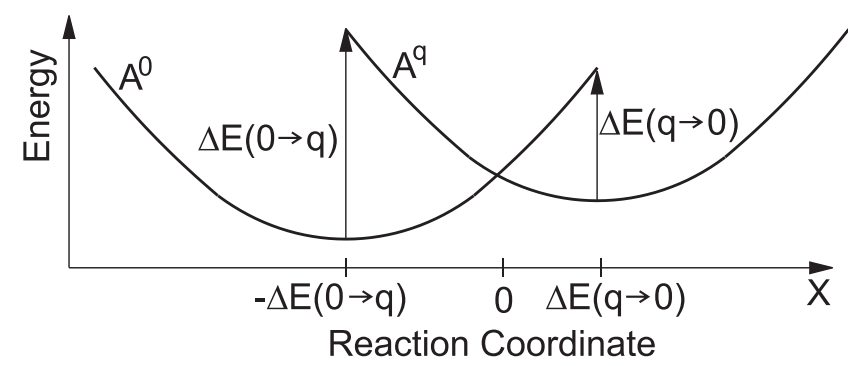

FIG. 4. Schematic energy diagram for the stabilization of a neutral adatom state $A^{0}$ and charged adatom state $A^{q}$ with charge $q e$ by ionic relaxation in a linear response model. The two diabatic potential energy curves, Marcus parabolas, are the minimum potential energies of $A^{0}$ and $A^{q}$ with respect to ionic configurations that are constrained to have a fixed value for the reaction coordinate $X$, defined as the potential energy difference between $A^{0}$ and $A^{q} . \Delta E(0 \rightarrow q)$ and $\Delta E(q \rightarrow 0)$ are the energy costs for charging $A^{0}$ and neutralizing $A^{q}$ at fixed ionic equilibrium configurations, respectively.

charged adatom states. A unique feature of Au is its large value of $2.3 \mathrm{eV}$ for $A$, which favors the stabilization of $\mathrm{Au}^{-}$, but the relatively small value of $1.3 \mathrm{eV}$ for $A$ of $\mathrm{Ag}$ shows that it is not a necessary condition for a stable negatively charged adatom state. However, this relatively small value for $A$ of $\mathrm{Ag}$ makes $\mathrm{Ag}^{-}$marginally stable: the increase of $\Phi$ for $\mathrm{NaCl}(2 \mathrm{ML}) / \mathrm{Cu}(111)$ compared with that for $\mathrm{NaCl}(2 \mathrm{ML}) / \mathrm{Cu}(100)$ and the decreasing electronic polarization from the metal substrate to $\Delta E_{\mathrm{el}}$ for increasing number of layers make $\mathrm{Ag}^{-}$unstable on $\mathrm{NaCl}(2 \mathrm{ML}) /$ $\mathrm{Cu}(111)$ and $\mathrm{NaCl}(3 \mathrm{ML}) / \mathrm{Cu}(100)$, respectively. Furthermore, the relatively small value for $\Phi$ of about $2.8 \mathrm{eV}$ for $\mathrm{NaCl}(2 \mathrm{ML}) / \mathrm{Cu}(311)$ tends to stabilize $\mathrm{A}^{-}$. In fact, only $\mathrm{Au}^{-}$was identified on $\mathrm{NaCl} / \mathrm{Cu}(311)$. The formation of $\mathrm{Ag}^{+}$is favored by its relatively small value for $I=7.6 \mathrm{eV}$ compared with $I=9.2 \mathrm{eV}$ for Au.

In conclusion, from scanning tunneling microscopy measurements and density functional theory calculations of $\mathrm{Ag}$ adatoms on $\mathrm{NaCl}$ bilayers supported by a $\mathrm{Cu}$ surface, we have demonstrated that charge multistability and the atomic-scale control of the charge state of adsorbates are not limited to $\mathrm{Au}$ adatoms and to bistability but that also tristability is possible. The key parameters behind the charge multistability are identified and suggest that it should be a common phenomenon, with important ramifications for molecular-scale electronics and memories.

Financial support from the EU Projects Nanoman, AMMIST, VR, and from the Volkswagen Foundation. Computer allocations by SNAC and discussions with Rolf Allenspach are gratefully acknowledged.

[1] X. H. Qiu, G. V. Nazin, and W. Ho, Phys. Rev. Lett. 92, 206102 (2004).
[2] J. Repp, G. Meyer, F. E. Olsson, and M. Persson, Science 305, 493 (2004).

[3] A. J. Heinrich, J. A. Gupta, C. P. Lutz, and D. M. Eigler, Science 306, 466 (2004).

[4] C. F. Hirjibehedin, C. P. Lutz, and A. J. Heinrich, Science 312, 1021 (2006).

[5] J. Repp, G. Meyer, S. Paavilainen, F. E. Olsson, and M. Persson, Phys. Rev. Lett. 95, 225503 (2005).

[6] S. W. Wu, G. V. Nazin, X. Chen, X. H. Qiu, and W. Ho, Phys. Rev. Lett. 93, 236802 (2004).

[7] M. Sterrer et al., J. Phys. Chem. B 110, 46 (2006).

[8] P. G. Piva et al., Nature (London) 435, 658 (2005).

[9] A. D. Miller et al., Science 297, 1163 (2002).

[10] M. Haruta, Catal Today 36, 153 (1997).

[11] G. Pacchioni, L. Giordano, and M. Baistrocchi, Phys. Rev. Lett. 94, 226104 (2005)

[12] N. Nilius, E. D. L. Rienks, H.-P. Rust, and H.-J. Freund, Phys. Rev. Lett. 95, 066101 (2005).

[13] As the stability of $\mathrm{Ag}^{-}$depends on its specific location with respect to the Moire pattern of the $\mathrm{NaCl} / \mathrm{Cu}(100)$ interface, only approximate values for the required manipulation voltages can be provided. The corresponding values depend only slightly on the current and refer to the low pA range.

[14] F. E. Olsson and M. Persson, Surf. Sci. 540, 172 (2003).

[15] W. Hebenstreit et al., Surf. Sci. 424, L321 (1999).

[16] F.E. Olsson, M. Persson, J. Repp, and G. Meyer, Phys. Rev. B 71, 075419 (2005).

[17] F.E. Olsson, M. Persson, A. G. Borisov, J. P. Gauyacq, J. Lagoute, and S. Fölsch, Phys. Rev. Lett. 93, 206803 (2004).

[18] L. Limot, E. Pehlke, J. Kröger, and R. Berndt, Phys. Rev. Lett. 94, 036805 (2005).

[19] G. Kresse and J. Furthmüller, Phys. Rev. B 54, 11169 (1996).

[20] The supercell (about $15 \times 15 \times 40 \AA^{3}$ ) contained a $\mathrm{Cu}$ slab with four layers of 36 atoms per layer, an adsorbed $\mathrm{NaCl}$ film with two layers of 32 atoms per layer, and a single Ag adatom. The surface Brillouin zone was sampled using $2 \times 2 k$ points and the plane wave cutoff energy was $300 \mathrm{eV}$. Ag adatoms in different initial local geometries at the $\mathrm{Cl}, \mathrm{Na}$, and bridge sites were geometrically optimized $(|F|<0.05 \mathrm{eV} / \AA)$, but only the most stable $\mathrm{Ag}^{0}, \mathrm{Ag}^{+}$, and $\mathrm{Ag}^{-}$sites are presented here.

[21] P. E. Blöchl, Phys. Rev. B 50, 17953 (1994).

[22] G. Kresse and D. Joubert, Phys. Rev. B. 59, 1758 (1999).

[23] J. P. Perdew et al., Phys. Rev. B. 46, 6671 (1992).

[24] J.P. Perdew, R. G. Parr, M. Levy, and J. L. Balduz, Jr., Phys. Rev. Lett. 49, 1691 (1982).

[25] V. I. Anisimov, F. Aryasetiawan, and A. I. Lichtenstein, J. Phys. Condens. Matter 9, 767 (1997).

[26] G. Makov and M.C. Payne, Phys. Rev. B 51, 4014 (1995).

[27] B. Li, A. Michaelides, and M. Scheffler, Phys. Rev. Lett. 97, 046802 (2006).

[28] The calculated adsorption energy and geometry of $\mathrm{Ag}^{0}$ adatom are basically the same for both the GGA and the GGA $+U$ functional.

[29] R. A. Marcus, Rev. Mod. Phys. 65, 599 (1993). 